\title{
Experimental Study on a Triple-base Propellant Formulation
}

\author{
CHEN Ming-lei ${ }^{1, a}$, MA Xiao, ${ }^{1, b}$, XIANG Kai-lun ${ }^{1, c}$, XING Ruo-ting $^{1, d}$, LIU \\ Wen-bin $^{1, \text { e }}$, ZHANG Bo ${ }^{1, f}$, WANG Na ${ }^{1, g}$, JI Dan-dan $^{2, h}$ \\ ${ }^{1}$ Gansu Province Yinguang Chemical Industry Group Co., Ltd,Baiyin 730900, China \\ ${ }^{2}$ School of Chemical Engineering,NUST,Nanjing 210094, China \\ achenml0611@163.com, ${ }^{b} \max 0522 @ 163 . c o m,{ }^{c} k a i l u n @ s i n a . c o m, ~{ }^{d}$ ruoting5677@sina.com, \\ eliu9866@sina.com, 'zhang9821@foxmail.com, ${ }^{\mathrm{g}}$ wangn78911@foxmail.com, ${ }^{\mathrm{h}}$ dandanji@163.com
}

Keywords: triple-base propellant, solvent ratio, formula, shrinking percentage

Abstract: In order to optimize the triple-base propellant's preparation technology and improve the combustion performance, used the half solvent method to prepare a series of propellant samples with different solvent ratios, and used the close bomb experiment and scanning electron microscope (SEM) and other experiments to study the microstructure, shrinkage rate and combustion performance. Results show that with the same formula but different solvent ratios, the shrinkage rate and the internal structure of the triple-base propellant were quite different, the solvent ratio 0.34 0.35 propellant's combustion performance is quite good and its formula was the best recipe.

\section{Introduction}

As the weapon of power energy, propellant is in the form of the combustion in the arms ${ }^{[1]}$. The burning propellant made this particular engine acquires heat and acting according to the law of the foundation. Therefore, combustion performance is not only one of the most important properties of gunpowder, but also directly affects the basic performance of the weapon system, such as muzzle velocity, range, accuracy and safety. Improving the preparation technology of the propellant is a important way to optimize the ballistic performance of the artillery, and also the important research direction of the propellant and the propellant charge technology $y^{[2-4]}$. Triple-base propellant is a kind of important propellant formulations; the preparation process is directly related to the combustion performance. In this paper, preparation of a triple-base propellant carried out exploration and research, compared to the different solvent ratio on the grain microstructure and combustion performance impact, thus obtains the optimum solvent ratio ${ }^{[5]}$.

\section{Experimental}

Materials and equipment. Nitrocellulose NC, Nitroglycerin NG, Nitro guanidine NGu, Acetone (AR, Shanghai Ling Feng Chemical Reagent Co. Ltd.), Ethanol (AR, Sinopharm Chemical Reagent Co. Ltd.).

Combustion performance test. Took the closed bomb test according to the GJB770B-2005, used the $200 \mathrm{ml}$ high pressure closed bomb, the experiment temperature was $20 \pm 2{ }^{\circ} \mathrm{C}$, packing density was $0.20 \mathrm{~g} / \mathrm{cm} 3$, ignition pressure was $10.98 \mathrm{MP}$, and the weight of the igniter charge bag which contain the dry nitrocellulose was about $2.0 \mathrm{~g}$. The frequency of the sampling systems was $1 \mathrm{MHz}$, designed FIR filter (used in the filtering program before tested data from the closed bomb) which the cut off frequency was $9940 \mathrm{~Hz}$; bandwidth was $2000 \mathrm{~Hz}$, stop band attenuation greater 
than $40 \mathrm{~dB}$. By testing the combustion static performance to calculate the $\Gamma-\Psi$ curve.

\section{Results and discussion}

Three-dimensional video display rearview mirror to observe the propellant surface, it can analyze the differences in the degree of kneading raw material and each sample within the pore size and thickness of the arc. The paper used microscope HiRox KH-1000 three-dimensional video microscope (Shenzhen Terry letter Electronics Co., Ltd.). Microscope images of the different solvent ratio sample following Figure 1, the measurement results in the pore size and thickness of the arc in Table 1.

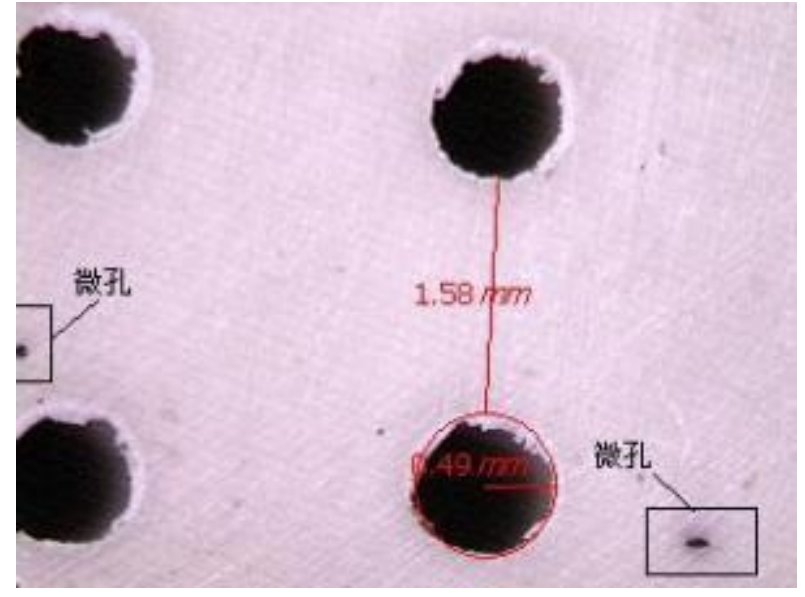

a) The solvent ratio 0.30 of the sample

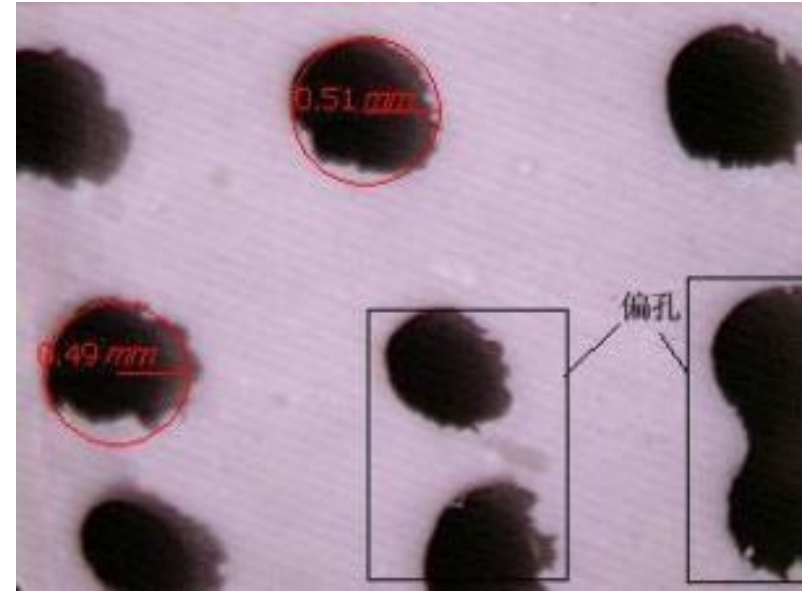

b) The solvent ratio 0.36 of the sample

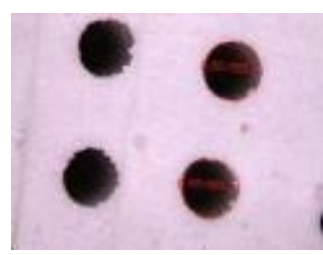

c) The solvent ratio 0.31 of the sample

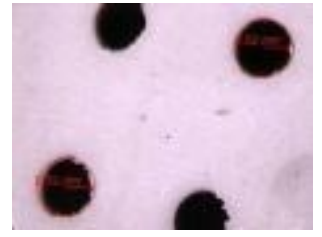
d) The solvent ratio
0.32 of the sample

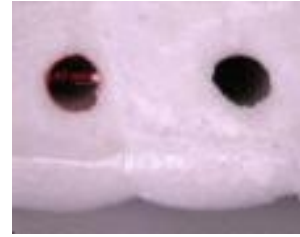

e) The solvent ratio 0.33 of the sample

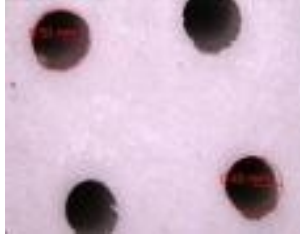

f) The solvent ratio

0.34 of the sample

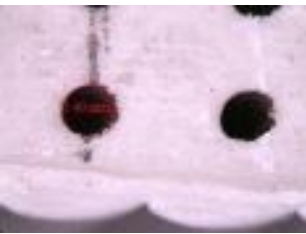

g) The solvent ratio 0.35 of the sample

Figure 1 Triple-base propellant three-dimensional video microscope photograph

Table 1 The average inner hole diameter and thickness arc of triple-base propellant

\begin{tabular}{cccccccc}
\hline Solvent ratio & 0.30 & 0.31 & 0.32 & 0.33 & 0.34 & 0.35 & 0.36 \\
\hline Inner hole diameter $/ \mathrm{mm}$ & 0.247 & 0.242 & 0.245 & 0.240 & 0.245 & 0.243 & 0.246 \\
thickness arc $/ \mathrm{mm}$ & 1.638 & 1.613 & 1.622 & 1.638 & 1.533 & 1.546 & 1.641 \\
\hline
\end{tabular}

Can be found in Figure 1, the seven different solvent ratio propellant samples' surface denseness were well, but the appearance of the sample which the solvent ratio of 0.30 had pores, and sample which solvent ratio of 0.36 had serious side hole, for which because the solvent is lower than the low level so that gelled, and solvent ratio is too high will make the original drug is too soft, the pressure stretching deformation process, eventually leading to partial hole finished propellant, porosity and thickness arc unequal results appear. It can be found that the size of the inner diameter of the arc thick and shrinkage associated, excluding the impact pressure drugs such as stretch and 
hand-cut thick and pore size of the arc caused by general law as propellant shrinkage greater the smaller the diameter, the greater the arc thick.

The different solvents of self-made triple-base propellant samples were selected randomly. Observed the microstructure by the SEM and magnified 2000 times. The microstructures of the observation are shown in figure 2 .

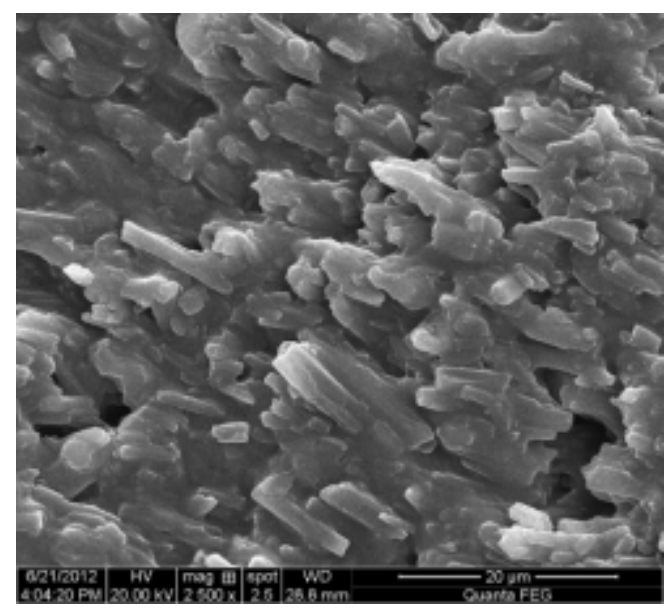

a) Nitroguanidine propellant' SEM photograph of

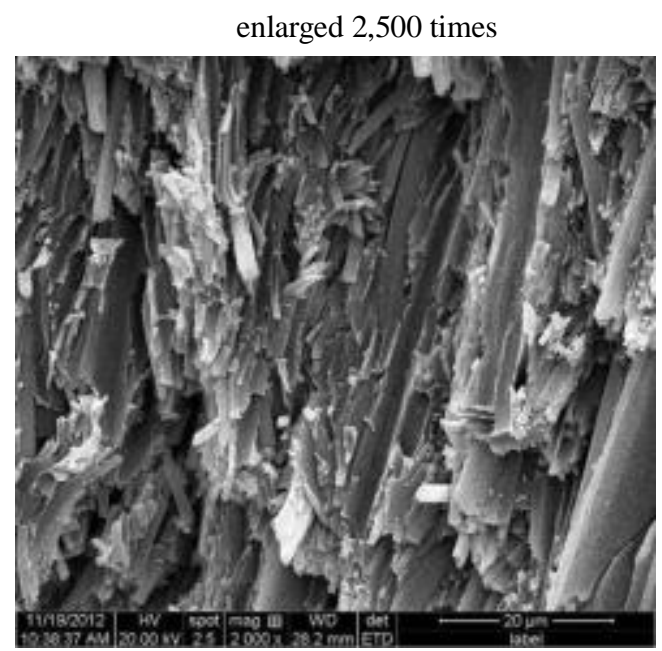

c) The solvent ratio 0.31 of the sample

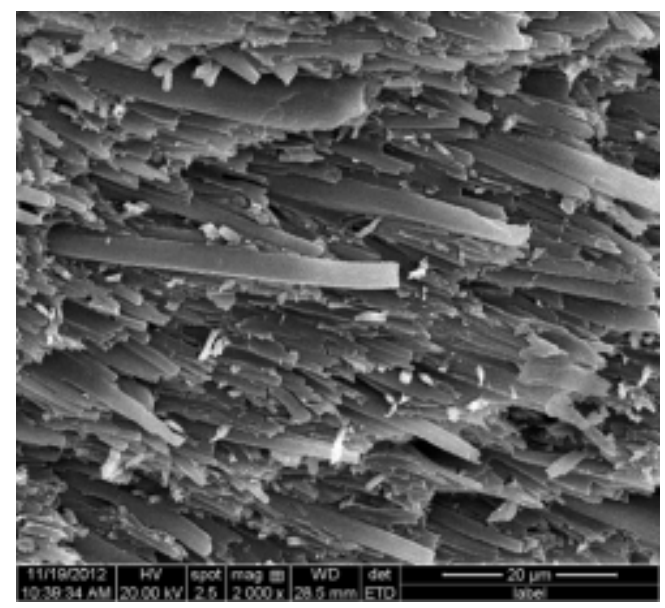

e) The solvent ratio 0.33 of the sample

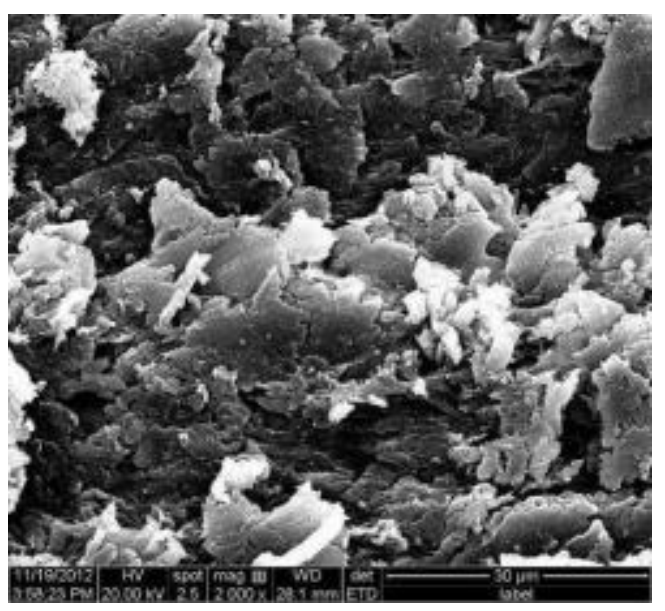

b) The solvent ratio 0.30 of the sample

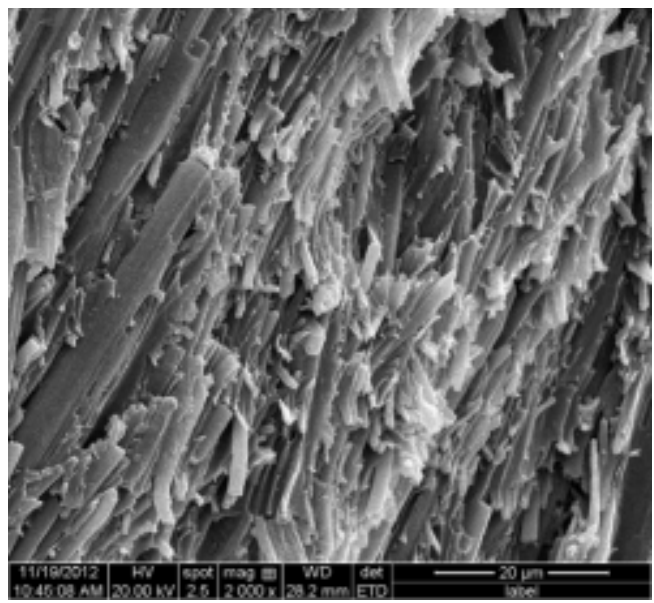

d) The solvent ratio 0.32 of the sample

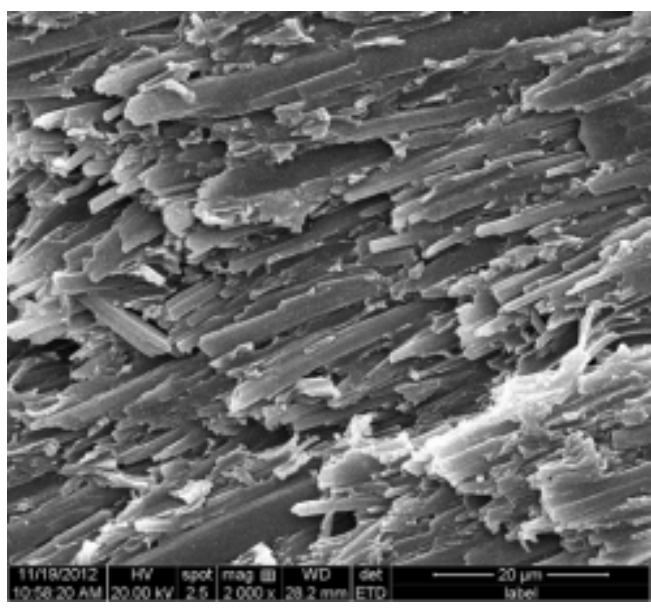

f) The solvent ratio 0.34 of the sample 


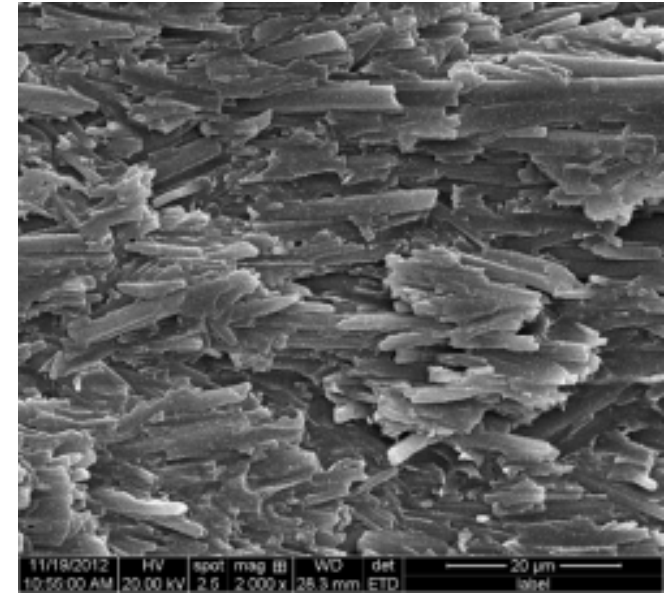

g) The solvent ratio 0.35 of the sample

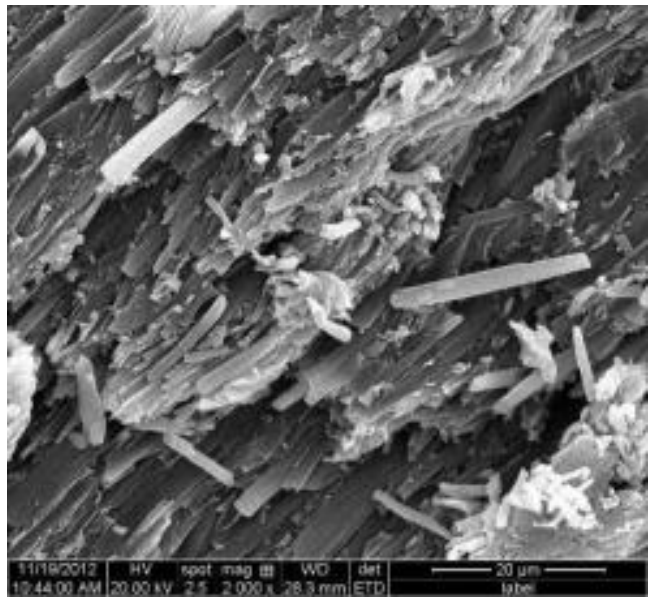

h) The solvent ratio 0.36 of the sample

Figure 2 Triple-base original propellants and seven different solvent ratio propellant samples surface scanning electron micrograph (2000 times)

Compare the above SEM photos which represent different solvent ratio types, it can be seen from the Fig 1 that the compactness of the solvent ratio 0.3 propellant is poor, the diameter of its hole is about 10 microns, internal structure is irregular, most are mixed and disorderly state of arrangement. May be it is due to the solvent ratio is so low that inadequate solvent in the process of kneading propellant had caused nitrocellulose and additives mixed unevenly.

As shown in Fig 2, the internal structure of the propellant sample which the solvent is 0.33 is better than the solvent ratio of 0.30 , but the sample shows the nitroguanidine arrangement is not neat.

It can be seen that the internal microstructures of specimens which the solvent ratio are 0.34 and 0.35 are better, the orientation arranges regularly, their orientation are along the propellant's extrusion direction (axis). The nitroguanidine arrangement of the sample which the solvent ratio is 0.34 is neat, deposition in the nitrocellulose, fully mixing dispersion evenly, but the nitroguanidine from the sample of solvent ratio 0.35 have shed partly.

\section{Closed bomb $\Gamma-\Psi$ curve.}

Usually use the $\Gamma-\Psi$ curve to analyze the process of actual combustion performance of propellant, $\Gamma$ is the propellant gas generated fierce degree, which can get from the explosive device test data processing. Calculated and drew the $\Gamma-\Psi$ curves of the four different solvent ratios of propellant, the specific conditions of the propellant combustion can be analyzed from the difference curves, thus provided a basis for improving the quality of the product. Different solvent ratios $\Gamma-\Psi$ curve are shown in figure 3. 


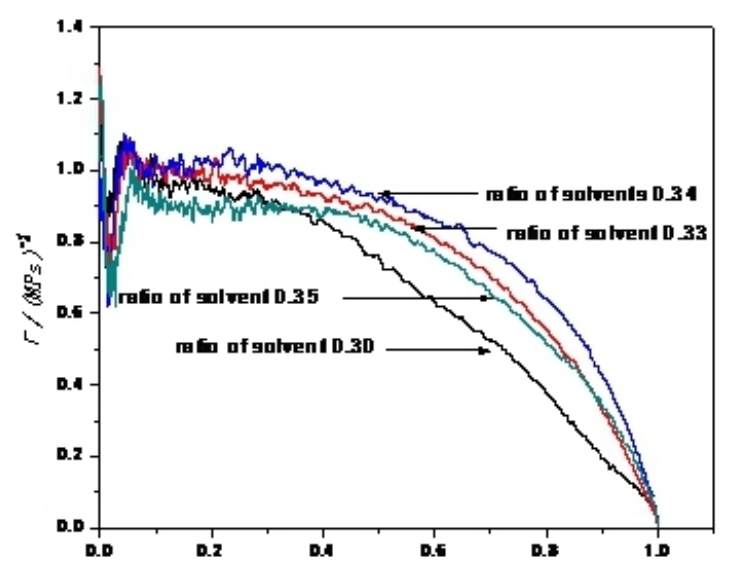

Fig 3 The $\Gamma$ - $\Psi$ curves of the four propellant samples

As shown in Fig 3, there is a significant difference of the four different solvent ratios propellant samples' $\Gamma-\Psi$ curves, the curve of the ignition gradually phase from the four segments is roughly the same. But in the last three stages of combustion, the value of $\Gamma$ of the solvent ratio 0.34 triple-base propellant has a greater value than the other three different samples, and has the best effect. The reason maybe is that the low solvent ratio cause the inner structure irregular, so that the samples are arranged messy state, and high solvent ratio (such as solvent ratio of 0.35) makes internal nitroguanidine shed partly. These reasons caused the differences of the pore sizes, leading to erosion caused by aerodynamic forces of different combustion phenomenon.

The final size of the propellant is not equal to the mold which prepares it. When the removal of solvents is complete, both the radial and axial of the propellant have different degree of contraction, the axial shrinkage is small and negligible, radial shrinkage is larger, must be taken into account. Before processing the propellant, it is must to predict shrinkage rate correctly, thus design the appropriate size of the mold ${ }^{[6]}$.

Shrinking percentage:

$$
C=\frac{D_{2}-D_{1}}{D_{2}} \times 100 \%
$$

In the formula: $\mathrm{D}_{2}$ is the model dimension, $\mathrm{D}_{1}$ is the final dimension.

In guarantee the preparation process is the same, only change the solvent ratio, made of four different solvent ratio propellants, and measured the sizes every $24 \mathrm{~h}$, from the start time to 3 days were to dry naturally, $4 \sim 5$ days for wet baking, $6 \sim 10$ days for drying baking. The final shrinkage rates comparison are shown in table 2 . 
Table 2 The shrinkage rates of the four different solvent ratio samples

\begin{tabular}{|c|c|c|c|c|}
\hline $\begin{array}{l}\text { Shrinking } \\
\text { percentage }(\%)\end{array}$ & 0.30 & 0.33 & 0.34 & 0.35 \\
\hline Thickness $2 \mathrm{e}_{1}$ & 9.52 & 11.24 & 7.06 & 7.69 \\
\hline Length $2 \mathrm{c}$ & 9.58 & 10.12 & 6.03 & 6.42 \\
\hline
\end{tabular}

The shrinkage rate of propellant mainly depends on the composition of propellant, the quality of raw materials, geometric shape, size and manufacturing technological conditions. These factors change, the shrinkage rate of gunpowder will follow to change. Contrast the four different solvent ratio samples' shrinkage rates, it is can be found that the solvent ratio of 0.30 and 0.33 samples' shrinkage ratios are larger than the solvent ratio of 0.34 and 0.34 , and the solvent ratio 0.34 sample's shrinkage rate is minimal. The reason may be that low solvent ratio makes the propellant's internal structure messy, internal diameter and inner surface are not as flat as the high solvent ratio of samples relatively, and the large specific surface area led the more volatile solvent, so sample contraction degree increases. When the solvent ratio is 0.36 or higher, because the sample solvent content is too high, volatile solvent makes final shrinkage deformation also increased.

\section{Conclusions}

Observed the propellants' $\Gamma-\Psi$ curves, found that the solvent ratio 0.34 sample combustion performance is stable, burning progressivity is better than other samples. By measuring the size of the samples, and calculate the shrinkage rates of the different solvent ratio samples, and found that the shrinkage rate of the solvent ratio 0.34 sample is the lowest. To sum up, when prepare triple-base nitroguanidine propellant the best solvent ratios are 0.34 and 0.35 .

\section{References}

[1] Wang Ze-shan, Shi Xian-yang. Low temperature sensitivity charge launch [M]. National defense industry press, 2006.

[2] DU Ping, WANG Ze-shan, HE Wei-dong. Theoretical Calculation of Broken-hole Process of LTSC Coated-propellant[J]. Journal of Nanjing University of Science and Technology, 2011(05):717-721.

[3] Yang Jian. Increasing burning propellant charge research [D]. The 204 Research Institute of China Ordnance Industry Group, 2002

[4] Studies on Process Technology of High Progressive and large web NQ-based Gun Propellant. Ph.D.Dissertation. Nanjing University of Science \& Technology. 2009.10.

[5] Wang Ze-shan. Gunpowder experiment method [M]. Beijing: Weapon Industry Press, 1996:84-85.

[6] Jin Zhi-ming. Interior ballistics of guns [M]. Beijing: Beijing Institute of Technology, 2007. 